\title{
Simulation of Natural Convection in a horizontal channel with heat sources mounted with porous blocks by the lattice Boltzmann method (MRT-LBM)
}

\author{
Kaoutar BOUARNOUNA ${ }^{1}$ *, Abdelkader BOUTRA ${ }^{1}$, Mahdi BENZEMA ${ }^{1}$, Mohammed El Ganaoui ${ }^{2}$, Youb Khaled \\ BENKAHLA $^{1}$ \\ ${ }^{1}$ Laboratoire des Phénomènes de Transfert, FGMGP, USTHB, Bab Ezzouar, Alger, Algérie. \\ ${ }^{2}$ University of Lorraine (Fédération Jacques Villermaux/LERMAB), Longwy Institute of Technology, France
}

\begin{abstract}
In this paper, laminar natural convection in a horizontal channel provided with porous blocks periodically distributed on its lower adiabatic surface has been analyzed. This numerical study is based on the multiple-relaxation-time (MRT) Lattice Boltzmann method (LBM). The two-dimensional model D2Q9 is adopted to solve the flow field, while the D2Q5 model is applied to solve the temperature field. The objective of the study is to analyze the effect of the Darcy number (10-1 $\leq \mathrm{Da} \leq 10-6)$, Rayleigh number $(103 \leq \mathrm{Ra} \leq 107)$ and the relative porous blocks height $(1 / 8 \leq \mathrm{D} \leq 1 / 2)$. The obtained results show the important effect of these parameters, which cannot be neglected, on both flow and the heat transfer structure, within this kind of channels.
\end{abstract}

\section{Nomenclature}

Da Darcy number $(=K / H 2)$

$d$ dimensional thickness of the porous substrate,

[m]

D dimensionless thickness of the porous substrate

$(d / H)$

$f i \quad$ density distribution function

$g \quad$ acceleration of gravity, [m $s-2]$

$H \quad$ channel height, [m]

$\mathrm{Nu} \quad$ local Nusselt number

$\mathrm{Pr} \quad$ Prandtl number $(=v / \alpha)$

$K \quad$ permeability of the porous medium (m2)

Ra Rayleigh number

$T$ temperature, $[K]$

$u \quad x$-velocity component, [m.s-1]

$v \quad y$-velocity component, [m.s-1]

$x, y \quad$ cartesian coordinates, $[\mathrm{m}]$

\section{Greek symbols}

$\begin{array}{ll}\alpha & \text { thermal diffusivity, }(=k /(\rho C p)) \\ & \begin{array}{l}\text { porosity of porous medium } \\ \text { kinematic viscosity, }[\mathrm{m} 2 . \mathrm{s}-1]\end{array} \\ \rho & \text { fluid density, [kg.m-3] }\end{array}$

Subscripts

$\begin{array}{ll}c & \text { cold } \\ h & \text { hot } \\ i & \text { direction index. }\end{array}$

\section{Introduction}

The study of convective heat transfer through porous media has long been of interest to researchers because of its presence in various industrial processes such as, solar receiver devices, ground water-hydrology building thermal insulation, heat exchangers, cooling problems in electronic systems, turbine blade cooling, energy storage units, drying processes, petroleum reservoir and geothermal operations. Given the complexity of these configurations, the numerical approach is very often used to solve the general equations governing flow and heat transfer.

Numerous numerical approaches have been developed over the last few years to simulate the different types of convective fluid flows, among which, Lattice Boltzmann method, noted by the abbreviation LBM, which is one of the most powerful and widely used methods, for the numerical resolution of flow and heat transfer problems in porous media due to its simplicity and ability to handle complex geometry and boundary conditions [1$6]$.

Into a porous space, (or a porous portion), the convection phenomenon has attracted the attention of many researchers all over the world, as the flow into this kind of spaces was very mysterious. The literature shows that this kind of investigation was dedicated to the free and forced convection ones. In their work, Ould-Amer et al. [7] have studied the advantage of using porous partitions to cooling electronic components found in a twodimensional horizontal channel. Guo and Zhao [8] have

\footnotetext{
* Corresponding author: bouarnouna2014@gmail.com
} 
developed a new numerical model to predict the incompressible fluid flow into a porous medium. Shokouhmand and al. [9] have treated the laminar flow of a Newtonian fluid within a totally and partially porous channel. El Ganaoui and Djebali [10] have simulated flow transitions using lattice-Boltzmann method D. Mouhtadi et al [11] have studied natural convection in a horizontal channel provided with heat generating blocks. Vahl Davis [12] have investigated numerically the natural convection of air in a square cavity a bench mark numerical solution. although, Seta and al. [13] have examined the Lattice Boltzmann simulation of natural convection phenomenon into a porous medium.

Motivated by these works, this paper a numerical study of laminar natural convection in a horizontal channel provided with porous blocks periodically distributed on its lower adiabatic surface on the fluid flow and heat transfer of a Newtonian fluid within a horizontal channel. The numerical study is developed using the Lattice Boltzmann method with multiple times of relaxation (MRT-LBM).

Keywords: Channel/ Porous Blocks/ Natural convection/ Lattice Boltzmann Method (LBM-MRT).

\section{Problem statement Mathematical formulation}

and

The studied configuration, shown in Fig. 1, consists of a two-dimensional channel with a height $(\mathrm{H})$, whose upper wall is maintained at a constant cold temperature. the channel is assumed infinite and comprising an infinite number of porous blocks periodically distributed on its lower wall. the blocks are mounted on the heat sources, a constant temperature $\mathrm{Th}=1$, the air $(\mathrm{Pr}=0.72)$ is used as heat transfer fluid. Because of the periodic nature of the problem and the imposed boundary conditions, the numerical calculations are restricted to the representative elementary domain of Fig. 2. The latter is limited by the fictitious planes P1 and P2 such that it contains two halves of contiguous blocks [11]

The boundary conditions associated to this problem are as follows:

- Periodicity conditions are applied on the fictitious planes $\mathrm{P} 1$ and $\mathrm{P} 2$.

- $\mathrm{Tc}=0$ on the upper channel surface and $\partial \mathrm{T} / \partial \mathrm{n}=0$ on the lower adiabatic surface.

- For the pores-air interface, the continuity of the temperature is imposed

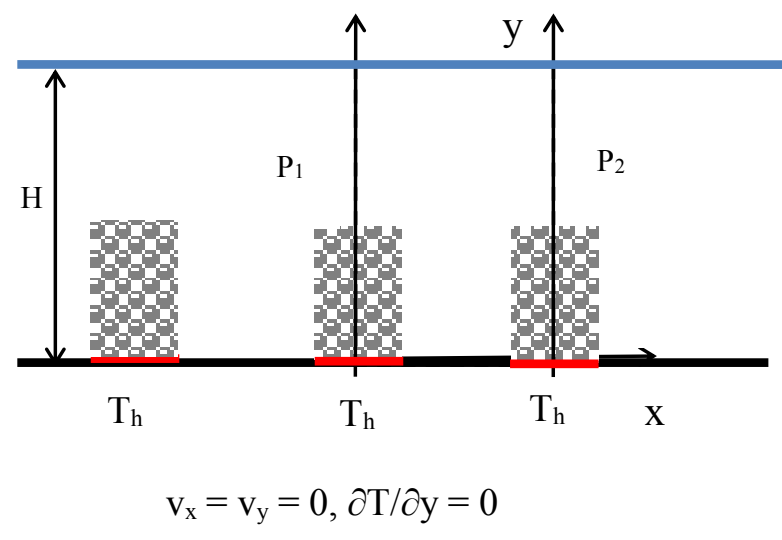

Fig.1: Physical problems scheme with its boundary conditions

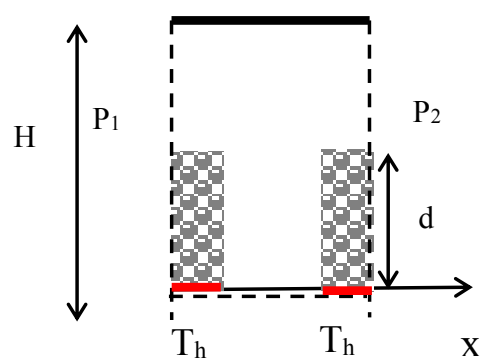

Fig. 2: Models used

The fundamental equations governing the fluid-porous system, in dimensionle form, are as follows:

Continuity equation:

$\frac{\partial v_{x}}{\partial x}+\frac{\partial v_{y}}{\partial y}=0$

$\mathrm{x}$ momentum equation:

$\frac{\partial v_{x}}{\partial t}+\frac{v_{x}}{\varepsilon} \frac{\partial v_{x}}{\partial x}+\frac{v_{y}}{\varepsilon} \frac{\partial v_{x}}{\partial y}=-\frac{\varepsilon}{\rho_{0}} \frac{\partial p}{\partial x}+v_{e}\left(\frac{\partial^{2} v_{x}}{\partial x^{2}}+\frac{\partial^{2} v_{x}}{\partial y^{2}}\right)+F_{x}$

y momentum equation:

$\frac{\partial v_{y}}{\partial t}+\frac{v_{x}}{\varepsilon} \frac{\partial v_{y}}{\partial x}+\frac{v_{y}}{\varepsilon} \frac{\partial v_{y}}{\partial y}=-\frac{\varepsilon}{\rho_{0}} \frac{\partial p}{\partial y}+v_{e}\left(\frac{\partial^{2} v_{y}}{\partial x^{2}}+\frac{\partial^{2} v_{y}}{\partial y^{2}}\right)+F_{y}$

Energy equation:

In the fluid region:

$\rho_{f} c_{p f}\left(\frac{\partial T}{\partial t}+v_{x} \frac{\partial T}{\partial x}+v_{y} \frac{\partial T}{\partial y}\right)=k\left(\frac{\partial^{2} T}{\partial x^{2}}+\frac{\partial^{2} T}{\partial y^{2}}\right)$

In the porous medium: 


$$
\rho_{f} c_{p f}\left(\sigma \frac{\partial T}{\partial t}+v_{x} \frac{\partial T}{\partial x}+v_{y} \frac{\partial T}{\partial y}\right)=k_{e}\left(\frac{\partial^{2} T}{\partial x^{2}}+\frac{\partial^{2} T}{\partial y^{2}}\right)
$$

In which $\rho_{0}$ is the mean fluid density, $\mathrm{v}, \mathrm{T}$ and $\mathrm{p}$ are the volume averaged fluid velocity, temperature, and pressure, respectively, is the porosity, $v_{\varepsilon}$ is the effective kinetic viscosity, the coefficient $\sigma=\left[\varepsilon\left(\rho_{\mathrm{f}} \mathrm{c}_{\mathrm{pf}}\right)+(1-\varepsilon)\left(\rho_{\mathrm{s}} \mathrm{c}_{\mathrm{ps}}\right)\right] / \rho_{\mathrm{f}} \mathrm{c}_{\mathrm{pf}}$ is the thermal capacity ratio, in which $\rho_{\mathrm{f}}\left(\rho_{\mathrm{s}}\right)$ and $\mathrm{c}_{\mathrm{pf}}\left(\mathrm{c}_{\mathrm{ps}}\right)$ are the density and specific heat of the fluid (solid) phase, respectively; $k\left(k_{e}\right)$ is the thermal conductivity of the fluid (porous medium) $\mathrm{F}$ $=(\mathrm{Fx}, \mathrm{Fy})$ denotes the total body force induced by the fluid-porous and other external forces, which can be expressed as [14-17].

$F=-\xi\left(\frac{\varepsilon v}{K} \mathrm{v}+\frac{\varepsilon C_{F}|\mathrm{v}| \mathrm{v}}{\sqrt{K}}\right)+\varepsilon\left(g \beta\left(T-T_{h}\right) j\right)$

Where $\mathrm{K}$ is the permeability, $v$ is the kinetic viscosity of the fluid, $\mathrm{g}$ is the gravitational acceleration, $\beta$ is the thermal expansion coefficient and $j$ is the unit vector in the y-direction and $\varepsilon$ is the porosity. In the present paper, the fluid-occupied region $\varepsilon=1.0$ is identified by setting the index $\xi=0$, and the porous region is tagged by $\xi=1.0$. The inertial coefficient $\mathrm{C}_{\mathrm{F}}$ and the permeability $\mathrm{K}$ of liquid flow through a porous medium with randomly distributed spherical particles can be expressed as [14,17].

$$
C_{F}=\frac{1.75}{\sqrt{175 \varepsilon^{3}}}, \quad K=\frac{\varepsilon^{3} d_{m}^{2}}{175(1-\varepsilon)^{2}},
$$

\subsection{Lattice Boltzmann approach}

The lattice Boltzmann scheme describes the distribution of particles $f_{j}\left(x_{i}, t\right)$ in $x_{i}$, of velocity $v_{j}$ at time $t$. In each node of the network, it's have $(j+1)$ distributions $f_{j}$.

In order to model the two-dimensional velocity field in the considered domain, the model D2Q9 on a square grid, of step $\Delta x=\Delta y=1$ was applied. The fluid particles move from one node of the gate to the neighboring node with discrete velocities, data ej [6]:

$$
\begin{aligned}
& e_{0}=(0,0) \\
& e_{1}=(1,0) ; e_{2}=(0,1) ; e_{3}=(-1,0) ; e_{4}=(0,-1) \\
& e_{5}=(1,1) ; e_{6}=(-1,1) ; e_{7}=(-1,1) ; e_{8}=(1,-1)
\end{aligned}
$$

The thermal field, meanwhile, has been described according to the model D2Q5 (network of five discrete temperatures).
The evolution of the medium in a time step $\Delta \mathrm{t}$ is broken down into two basic stages:

collision and advection. These two steps are described by the following equation:

$f_{j}\left(x+v_{j} \Delta t, t+\Delta t\right)=f_{j}(x+t)+\Omega_{j} f_{j}(x+t)+C_{j}$

Where $f_{j}$ is the distribution function of a particle, $\Omega$ is the collision operator, representing the variation of the distribution function due to particle collisions and $\mathrm{C}_{\mathrm{j}}$ is the forcing term.

The linearization of this operator around the local equilibrium distribution function brings a significant simplification of the LBM method

On each domain node, the nine moments associated with the nine distribution functions are linked by the following linear transformation [18]:

$m=M . f$

where the matrix $\mathrm{M}$ is of order 9. During the colliding step which is local in space, three moments are preserved (the density and the momentum, in the longitudinal direction and the transversal direction), the six remaining moments, not conserved, are calculated from a simple equation of linear relaxation towards the equilibrium values which depend on the conserved quantities [18]:

$m_{k}^{*}(x, t)=\left(1-s_{k}\right) m_{k}(x, t)+s_{k} m_{k}^{e q}+C_{k}$

$\mathrm{s}_{\mathrm{k}}=\Delta \mathrm{t} / \tau$ being the relaxation rate, $\tau_{\mathrm{k}}$ the relaxation time, $\mathrm{m}_{\mathrm{k}}$ the moment after collision and $\mathrm{m}^{\mathrm{eq}}$ is the equilibrium moment value.

For a reason of stability, relaxation rates verify double inequalit $0 \leqslant s k \preccurlyeq 2$.

$v=\frac{1}{3}\left(\frac{1}{s_{8}}-\frac{1}{2}\right)$

The new distribution functions $\mathrm{f}^{*}$ are calculated from the new moments $\mathrm{m}^{*}$ :

$f^{*}=M^{-1} m^{*}$

On the Macroscopic scale, the mass density and the speed vector are given as follows $[6,18]$ :

$\rho=\sum_{j=0}^{8} f_{j}(x, t)$

$\rho_{0} \mathrm{v}\left(x_{j}, t\right)=\sum_{i=0}^{8} e_{i} f_{i}\left(x_{j}, t\right)+\frac{\delta}{2} \rho_{0} F$ 
The heat transfer between the wall of the canal and the fluid is characterized by the Nusselt number $\mathrm{Nu}$ and average $\mathrm{Nu}_{\text {avg }}$ [19]:

$$
N u=\left.\frac{2 H}{T_{m}-T_{c}} \frac{\partial T}{\partial y}\right|_{y=0}, N u_{m o y}=\sum \frac{1}{x_{2}-x_{1}} \int_{x_{1}}^{x_{2}} N u d x
$$

Where $T_{m}$ is the average mixing temperature:

$$
T_{m}=\frac{\int_{0}^{H} u T d y}{\int_{0}^{H} u d y}
$$

\section{Code validation}

In order to verify the reliability of the numerical results obtained in the present work, a validation of numerical simulations was made, comparing them with those of the literature.

A first comparison with a work investigated by Vahl Davis [12] is undertaken. The latter consider a numerical study dealing with the natural convection of air within a cavity, defferentially heated, where the $\mathrm{Nu}_{\text {moy }}$ transfer rate (Table 1) is compared with those of Vahl Davis [12]

A second comparison of the results was made with those of Mouhtadi et al [11], for which, the phenomenon of natural convection in a horizontal channel provided with heat generating blocks.l. The numerical results are shown in Figures 3 (a) and 2 (b)

A third comparison with those of. Sung et al. [20], for which, the phenomenon of convection takes place in a channel with adiabatic walls. A rectangular porous obstacle is mounted on the hot springs, which are located at the lower wall of the channel. The numerical results are shown in Figures 3 (c) and 3 (d).

It's was noted that results are in very good agreement with those mentioned previously, and the relative differences do not exceed $1.3 \%$, which allows us to confirm the validity of the method used

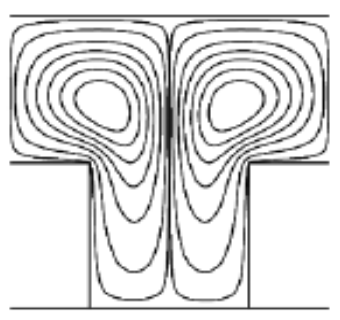

(a) Mouhtadi et al [11]

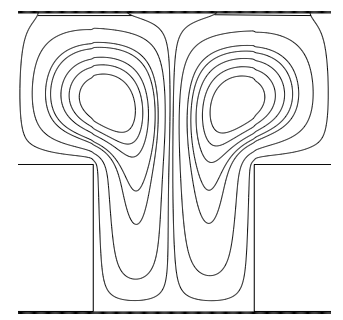

(a) Present prediction
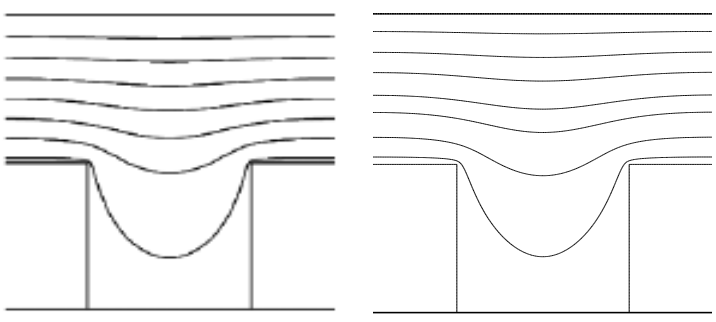

(b) Mouhtadi et al [11]

(b) Present prediction
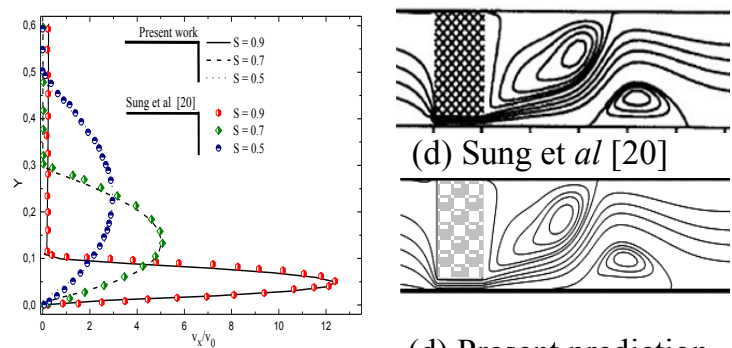

(d) Present prediction

(c) Velocity profiles

Fig. 3:

The results compared with the other literatures: (a) and (b) streamlines and isotherms compared with Mouhtadi et $a l$ for $\mathrm{Ra}=10^{4}, \mathrm{Pr}=0.71, \mathrm{k}^{*}=\mathrm{k}_{\mathrm{s}} / \mathrm{k}_{1}=$ 200. (c) and (d) Velocity profiles and streamlines compared with Sung et al [20] for $\mathrm{Re}=100, \mathrm{Da}=$ $10^{-5}$.

Table 1: Comparison of the average number of

\begin{tabular}{|c|c|c|c|c|}
\hline & $\mathrm{Ra}$ & $10^{4}$ & $10^{5}$ & $10^{6}$ \\
\hline & Vahl Davis [12] & 2.242 & 4.564 & 9.270 \\
\hline $\mathrm{Nu}_{\mathrm{avg}}$ & $\begin{array}{c}\text { present numerical } \\
\text { results }\end{array}$ & 2.251 & 4.613 & 9.301 \\
\hline & $\begin{array}{c}\text { Relative } \\
\text { difference } \%\end{array}$ & 0.4 & 1.1 & 0.33 \\
\hline
\end{tabular}
Nusselt with those of Vahl Davis [12] for $\operatorname{Pr}=0.71$.

In order to check the mesh independence of the results, a grid convergence study is performed for the convection phenomenon inside the squares containing porous blocks previously shown in Fig. 2 with $\operatorname{Pr}=0.71, \mathrm{Ra}=10^{3}, \mathrm{D}=$ 0.5 . Several mesh distributions ranging from $81^{2}$ to $401^{2}$ were tested. the average Nusselt number versus grid, are shown in Figure 4. It is observed $201^{2}$ uniform grid is adequate for a grid independent solution for different Darcy numbers. 


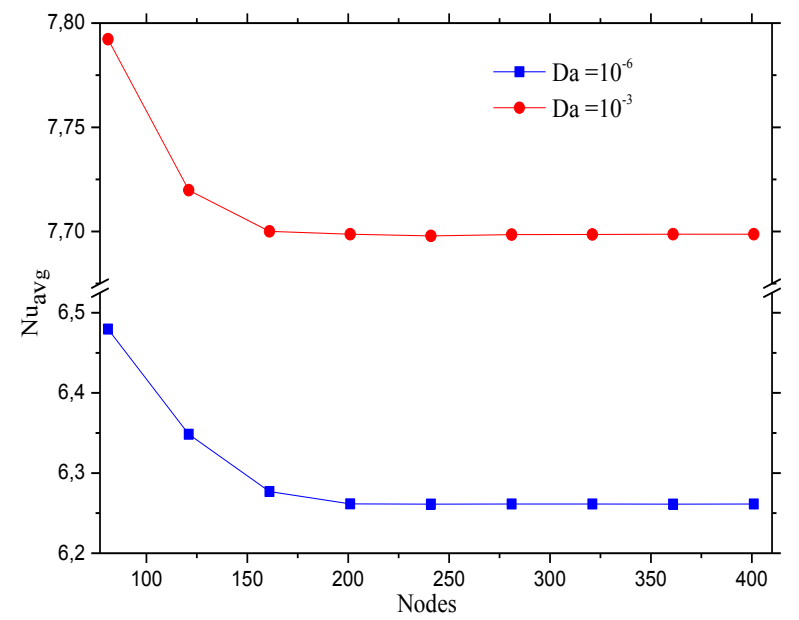

Fig. 4: Averaged Nusselt number of the porous blocks for several uniform grids, $\mathrm{Ra}=10^{3}, \mathrm{D}=0.5$.

\section{Results and discussion}

In this section the numerical results are discussed in order to study the effects of the Darcy number Da, Rayleigh number $\mathrm{Ra}$ and the relative blocks height $\mathrm{D}$ with $\mathrm{Pr}=0.71$. The effect of $\mathrm{Da}$ on streamlines and isotherms is shown in Figure 5. It is clear from this figure that the growth of $\mathrm{Da}$ of $10^{-3}$ to $10^{-1}$ does not lead to significant changes in the isolines of stream function and temperature the increase of $\mathrm{Da}$ is related to a growth of a permeability of the porous obstacles, the fluid arrives to penetrate the porous obstacles without disturbance nor important braking and thus the flow patterns will be that of the corresponding free convection problem with no porous medium. On the other hand, when $\mathrm{Da}$ decreases by $10^{-4}$ to $10^{-5}$, the resistance to flow caused by the presence of the porous medium increases. This causes the decrease of the fluid velocity in the channel. The increase of the Darcy number of obviously modifies the movement of the isotherms in the chanal when they cross more distance. In addition, their density on the hot wall phenomenally improves which causes the increase of the temperature of the gradient on the hot wall. Therefore, it demonstrates the increase in heat transfer by improving Darcy number. Figure 6 describes the effects of the Rayleigh number for variations relative porous blocks height on the average Nusselt number. As it can be seen from this figure, the average Nusselt number generally increases with increasing the Rayleigh number, i.e. as the heat transfer regime becomes convection-dominated. For $\mathrm{Ra} \leq 10^{4}$, the average Nusselt number remains nearly constant . In that case, the heat transfer regime is conductiondominated. Moreover, the values of average Nusselt number increases slowly with the increasing of relative porous blocks height for lower values of $\mathrm{Ra} \leq 10^{4}$ and increases rapidly for $\mathrm{Ra}>10^{4}$. The greatest heat transfer rate is found for the highest value of the Rayleigh number and relative porous blocks height $D=1 / 2$. Figure 7 illustrates the average Nusselt number for various Darcy number and relative porous blocks height .The average Nusselt number shows that the heat transfer declines with the rise of relative porous blocks height generally. Furthermore, it demonstrates that the drop of Darcy number decreases heat transfer markedly.

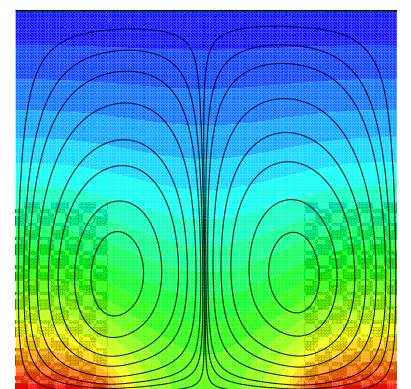

$\mathrm{Da}=10^{-1}$

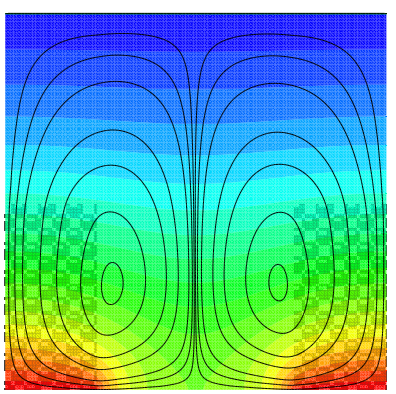

$\mathrm{Da}=10^{-3}$

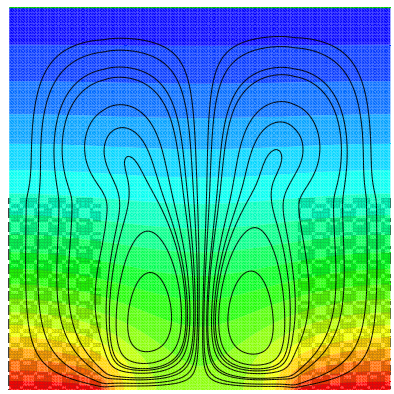

$\mathrm{Da}=10^{-5}$

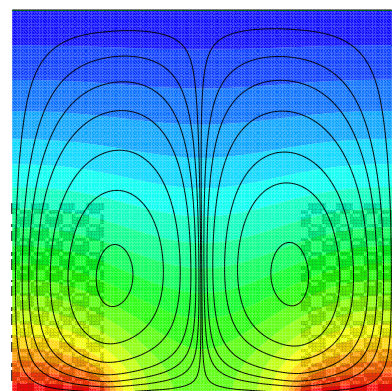

$\mathrm{Da}=10^{-2}$

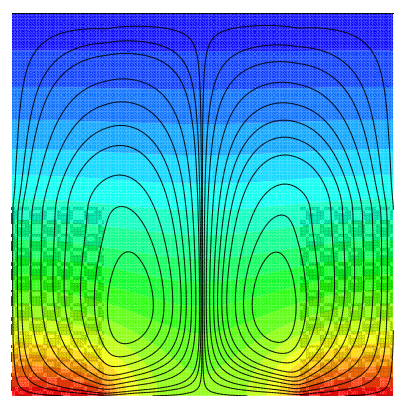

$\mathrm{Da}=10^{-4}$

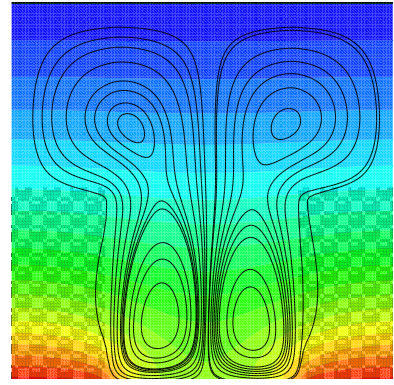

$\mathrm{Da}=10^{-6}$
Fig.5: streamlines and isotherms for various Darcy number for $\mathrm{Ra}=10^{4}, \mathrm{D}=1 / 2$.

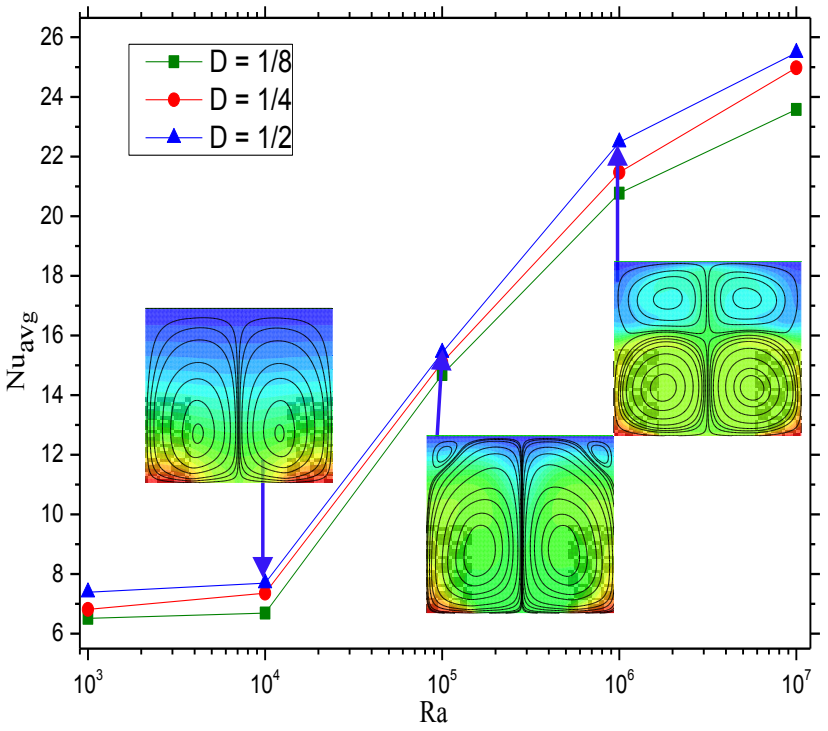

Fig. 6: Effects of the Rayleigh number for variations relative porous blocks height $\mathrm{D}$ on the averaged local Nusselt number for $\mathrm{Da}=10^{-3}$. 


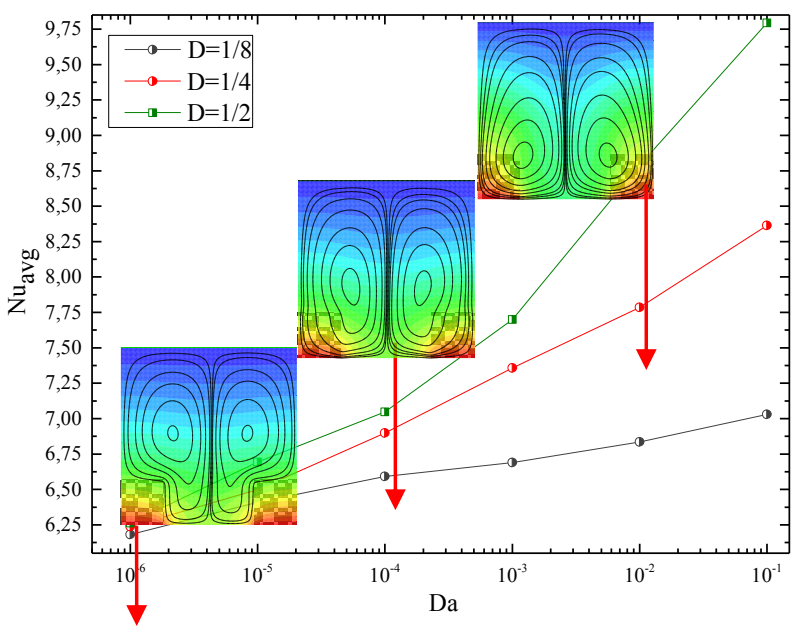

Fig.7: Effects of the Darcy number for variations relative porous blocks height $\mathrm{D}$ on the averaged Nusselt number. $\mathrm{Ra}=$ $10^{4}$.

\section{Conclusion}

Naturel convection in a horizontal channel provided with porous blocks periodically distributed on its lower adiabatic surface has been conducted by a double multiple-relaxation-time lattice Boltzmann method. This simulation has been performed for the pertinent parameters in the following ranges: Darcy number $\left(10^{-1}\right.$ $\left.\leq \mathrm{Da} \leq 10^{-5}\right)$, Rayleigh number $\left(10^{3} \leq \mathrm{Ra} \leq 10^{7}\right)$ and the relative porous blocks height $(1 / 8 \leq \mathrm{D} \leq 1 / 2)$. This investigation is analyzed for various mentioned parameters and the following results were acquired.

The good agreement with previous numerical investigations demonstrates that the lattice Boltzmann method (MRT-LBM) is an appropriate method for the studied problem.

Heat transfer increase with an increase in Rayleigh number generally for different studied parameters.

The greatest heat transfer rate is found for the highest value of relative porous blocks height $\mathrm{D}=1 / 2$.

The drop of Darcy number $\left(\mathrm{Da} \leq 10^{-4}\right)$ decreases heat transfer markedly.

\section{References}

1. C.K. Aidun, and J.R. Clausen, Lattice-Boltzmann method for complex flows, Annu. Rev. Fluid Mech, 42, pp. 439-472 (2010).

2. W.H. Zhang, Z.H. Chai, B.C. Shi B.C, Z.L. Guo, Lattice Boltzmann study of flow and mixing characteristics of two-dimensional confined impinging streams with uniform and non-uniform inlet jets, Comput.Math.Appl, 65,pp. 638-648 (2013).
3. Q. Liu, Y.L. He, Q. Li, W.Q. Tao, A multiplerelaxation-time lattice Boltzmann model for convection heat transfer in porous media, Int. J. Heat Mass Transf, 73, pp.761-781 (2014).

4. Z. Tao and D. Che, Double MRT thermal lattice Boltzmann simulation for MHD natural convection of nanofluids in an inclined cavity with four square heat sources (2016).

5. Y.T. Yang, S.C. Chang, C.S. Chiou, Lattice Boltzmann method and large-eddy simulation for turbulent impinging jet cooling. Int. J. Heat Mass Transf, 61, pp.543-554.

6. C,Y. Peng, Shu and Y.T. Chew, Simplified thermal lattice Boltzmann model for incompressible thermal flows, Phys. Rev. E 68 pp. 026-701, (2003)

7. Y. Ould-amer, S. Chikh,, G. Bouhadef, Lariat, Forced convection cooling Flow enhancement by using porous materials, Int. J. Heat Fluid Flow, pp, 251-258.

8. Z. Guo and T.S.Zhao, Lattice Boltzmann model for incompressible flowsthrough porous media, Phys. Rev. 66,pp, 306-304 (2002).

9. H. Shokouhmand, F. Jam M.R. Salimpou, Simulation of laminar flowand convective heat transfer in conduits filled with porous media using Lattice Boltzmann MethodInt. Communications in Heat and Mass Transf, 36,pp, 378-384 (2009).

10. M. El-Ganaoui and R. Djebali, Aptitude of a lattice Boltzmann method for evaluating transitional thresholds for low Prandtl number .ows in enclosures, Comptes Rendus Mécanique, 338, 85-96 (2010).

11. D. Mouhtadi, A. Amahmid, M. Hasnaoui, R. Bennacer, Natural convection in a horizontal channel provided with heat generating blocks:Discussion of the isothermal blocks validity, Energy Conversion and Management, 53 45-54 (2012)

12. G. De vahl davis, natural convection of air in a square cavity a bench mark numerical solution, international journal for numerical methods in fluids, vol. 3, 249-264 (1983).

13. T. Seta, E. Takegoshi, K. Okui, Lattice Boltzmann simulation of natural convection in porous media, Math.Comput.Sim, 72,pp. 195-200 (2006).

14. Z. Guo T.S. Zhao, A lattice Boltzmann model for convection heat transfer in porous media. Numer. Heat Transfer B 47:157-177 (2005).

15. C.T. Hsu, P. Cheng, Thermal dispersion in a porous medium. Int. J. Heat Mass Transfer 33 :1587-1597 (1990). 
16. C. Beckermann, R. Viskanta, Natural convection solid/liquid phase change in porous media, Int. J. Heat Mass Transfer 31:35-46 (1988).

17. W.J. Chang, D.F Yang, Natural convection for the melting of ice in porous media in a rectangular enclosure, Int. J. Heat Mass Transfer 39:2335-2348 (1996).

18. A. Mezrhab, M. Bouzidi, P. Lallemand, Hybrid lattice Boltzmann finite-difference simulation of convective flows, Computer and Fluids, 33,pp, 623-641 (2004).

19. A.A. Alamyane, A A. Mohamad, Simulation of forced convection in a channel with extended surfaces by the lattice Boltzmann method,Computers and Mathematics with Applications, 59, 2421-2430 (2010).

20. H.J. Sung, S.Y. Kim, Forced convection from an isolated heat source in a channel with porous medium, Int. J. Heat and Fluid Flow, 16 (1995). 